03

\title{
Влияние числа Рейнольдса на распределение пульсационной составляющей вихря скорости по сечению плоского канала
}

\author{
(C) Ю.Г. Чесноков
}

Санкт-Петербургский государственный технологический институт (технический университет), 198013 Санкт-Петербург, Россия

e-mail: ygchesnokov@yandex.ru

(Поступило в Редакцию 24 июля 2017 г. В окончательной редакции 25 сентября 2018 г.)

На основе анализа данных, полученных различными авторами методом прямого численного моделирования течения жидкости в плоском канале, изучено влияние числа Рейнольдса на распределение среднеквадратичных значений проекций пульсационной составляющей вихря скорости по сечению плоского канала.

DOI: $10.21883 /$ JTF.2019.03.47167.2444

\section{Введение}

Важнейшей характеристикой турбулентного потока является наличие завихренности. Однако экспериментальное измерение проекций вихря скорости представляет собой весьма сложную проблему [1]. Такого рода измерения проводились как для турбулентных течений в плоском канале [2], так и для турбулентных течений в пограничном слое [3-6]. Как отмечается в работе [7], измерения не являются достаточно точными, чтобы выявить влияние числа Рейнольдса на распределение завихренности по сечению канала. В особенности это относится к области вблизи твердой стенки. Наиболее детальную информацию можно получить при помощи данных, полученных методом прямого численного моделирования турбулентного течения в плоском канале [8-16]. Результаты прямого численного моделирования, полученные различными авторами, использовались в работах [17-19] для изучения влияния числа Рейнольдса на различные статистические характеристики течений. Если первоначально расчеты могли осуществляться лишь при небольших значениях числа Рейнольдса, то в работах [14-16] значение этого параметра (рассчитанное по средней скорости и эквивалентному диаметру канала) достигает примерно 50000.

Будем рассматривать турбулентное течение в плоском канале с полушириной канала $h$. Введем в рассмотрение декартову систему координат $(x, y, z)$, ось $x$ которой направлена по оси канала, ось $y$ по нормали к стенке, а ось $z$ по нормали к плоскости течения. Через $\omega_{x}, \omega_{y}$ и $\omega_{z}$ обозначим среднеквадратичные значения проекций пульсационной составляющей вихря скорости на оси $x$, $y$ и $z$ соответственно. Через $\omega$ обозначим среднеквадратичное значение модуля вектора пульсационной составляющей скорости: $\omega^{2}=\omega_{x}^{2}+\omega_{y}^{2}+\omega_{z}^{2}$. Величину $\omega^{2} / 2$ иногда называют энстрофией. Введенные в рассмотрение величины могут зависеть от расстояния у до стенки, напряжения трения на стенке $\tau$, физических характеристик жидкости (плотности $\rho$ и вязкости $\mu$ ) и полуширины канала $h$. Обозначим через $u_{r}=\sqrt{\tau / \rho}$ так называемую динамическую скорость жидкости, а через $l_{\tau}=v / u_{r}$ динамическую длину. Здесь $v=\mu / \rho-$ кинематическая вязкость жидкости. Если в качестве масштабов длины и скорости использовать динамическую длину и динамическую скорость, то соответствующие безразмерные переменные обычно называют внутренними и обозначают при помощи надстрочного индекса „плюс“. Из соображений размерности вытекает, что во внутренних переменных безразмерные величины $\omega_{x}^{+}, \omega_{y}^{+}$и $\omega_{z}^{+}$ будут зависеть только от двух безразмерных параметров, например, $y^{+}$и $\operatorname{Re}_{\tau}=u_{\tau} h / v=h^{+}$- числа Рейнольдса, рассчитанного по динамической скорости жидкости и полуширине канала. Эту величину называют также числом Кармана. Обычно считают, что в окрестности стенки полуширина канала оказывает малое влияние на закономерности течения. Тогда в этой области во внутренних переменных зависимости статистических характеристик турбулентности от $y^{+}$должны быть универсальными, т.е. не зависеть от числа $\operatorname{Re}_{\tau}$. Однако, как показывают результаты прямого численного моделирования [8-16], значения величин $\omega_{x}^{+}$и $\omega_{z}^{+}$при фиксированном значении $y^{+}$с ростом $\operatorname{Re}_{\tau}$ увеличиваются. Как показывает рис. 1, в пределах так называемого вязкого подслоя и буферной зоны зависимость $\omega^{2}$ от $\operatorname{Re}_{\tau}$ проявляется весьма сильно.

В работе [7] во внутренней области (при сравнительно малых $y^{+}$) для величин $\omega_{x}^{2}$ и $\omega_{z}^{2}$ и предлагается использовать асимптотические разложения, содержащие два слагаемых. Малым параметром является отношение $u_{\tau} / U_{0}$. Здесь $U_{0}-$ скорость на оси канала. В переменных стенки главные члены разложений пропорциональны $U_{0} / u_{\tau}$, а вторые слагаемые обращаются в нуль на стенке. Если это так, то при $y^{+}=0$ величина $u_{\tau} \omega^{+2} / U_{0}$ не должна зависеть от числа Рейнольдса $\operatorname{Re}_{\tau}$. Однако, как показывает рис. 2, наблюдается быстрое увеличение этой величины при сравнительно небольших значениях $\operatorname{Re}_{\tau}$. При больших значениях этого параметра имеются расхождения между результатами работы [15] и работ $[14,16]$. Согласно последним двум работам, ука- 


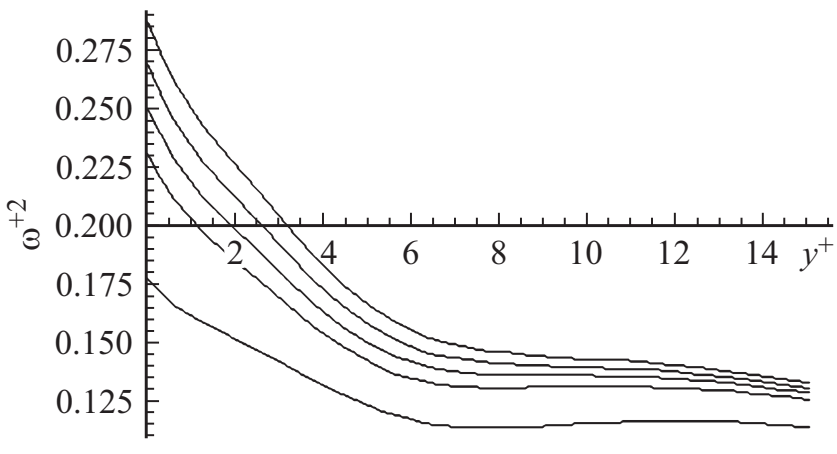

Рис. 1. Зависимости $\omega^{+2}$ от $y^{+}$при различных значениях $h^{+}$, построенные по результатам работы [16]. Значения $h^{+}$равны 182, 543, 1001, 1995 и 5186. Чем больше $h^{+}$, тем выше располагается соответствующая кривая.

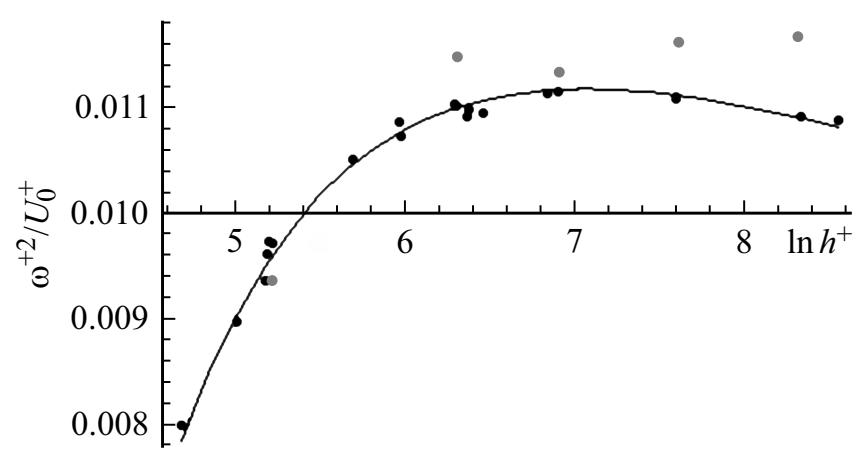

Рис. 2. Зависимость $\omega^{+2} / U_{0}^{+}$от $\ln h^{+}$. Черные точки построены по результатам работ $[8-14,16]$, серые - по результатам работы [15]. Кривая построена по формулам (1) и (2).

занная величина достигает максимального значения примерно при $h^{+}=1000$, а затем медленно убывает. Значения $u_{\tau} \omega^{+2} / U_{0}$, полученные по данным [15], оказываются более высокими. Отметим, что величина $U_{0}^{+}=U_{0} / u_{\tau}$ с ростом $h^{+}$увеличивается по логарифмическому закону. В работе [20] для ее расчета предложена следующая формула:

$$
U_{0}^{+}=\frac{1.000}{\ln h^{+}-3.886}+3.964+2.632 \ln h^{+} .
$$

Сплошная линия на рис. 2 построена при помощи аппроксимационной формулы

$$
\omega^{+2}=0.411-\frac{0.8546}{\ln h^{+}-1.57}
$$

и формулы (1). Коэффициенты в формуле (2) определены путем аппроксимации данных прямого численного моделирования, полученных в $[8-14,16]$.

Во внешней области в качестве масштаба длины используют полуширину канала $h$. При фиксированном значении $Y=y / h$ величины $\omega_{x}^{+}, \omega_{y}^{+}$и $\omega_{z}^{+}$с ростом $\operatorname{Re}_{\tau}$ убывают. Следует отметить, что во внешней области эти величины сравнительно слабо различаются между собой (см., например, [3]). На основании данных работ [10,11] в [7] найдено, что при $y=0.1$ величины $\omega_{i}^{+}$(где $i$ принимает значения $x, y$ и $z$ ) убывают примерно как $1 / \operatorname{Re}_{\tau}$. Об этом же свидетельствуют и экспериментальные данные (см., например, [21]). Анализ данных работ [8-16] показывает, что имеются определенные отклонения от указанных закономерностей.

Цель настоящей работы заключается в изучении закономерностей распределения проекций пульсационной составляющей вихря скорости в зависимости от расстояния до стенки как во внутренней, так и во внешней областях течения.

\section{Зависимость модуля пульсационной составляющей вихря скорости от расстояния до стенки во внешней области}

В той области, где справедлива логарифмическая формула для распределения скорости, вид зависимости $\omega^{+2}$ от $Y$ можно установить при помощи соображений, высказанных еще в книге Ландау и Лифшица [22]. Как отмечается в этой книге, пульсационная скорость величина порядка $u_{\tau}$ и поэтому с логарифмической точностью мала по сравнению с осредненным значением скорости $U$. Поэтому в уравнении баланса кинетической энергии турбулентных пульсаций диссипация энергии $\varepsilon$ в единице объема жидкости примерно равна слагаемому, описывающему обмен энергией между осредненным и пульсационным движением:

$$
\varepsilon=-\overline{u \nu} \frac{d U}{d y} .
$$

Здесь $u$ и $v$ - проекции пульсационной скорости на оси $x$ и $y$ соответственно, черта над величиной обозначает операцию осреднения. В первом приближении в так называемом логарифмическом пограничном слое $-\overline{u \nu}=u_{\tau}^{2}$, а $d U / d y=u_{\tau} /(\kappa y)$. Здесь $\kappa-$ так называемая постоянная Кармана. Для однородной турбулентности $\varepsilon=v \omega^{2}$. С хорошей степенью точности это соотношение будет выполняться и в логарифмическом пограничном слое. Поэтому $\omega^{2}=u_{\tau}^{3} /(\kappa v y)$. В безразмерной форме эта формула записывается так:

$$
h^{+} \omega^{+2}=\frac{1}{\kappa Y} .
$$

Как вытекает из этого соотношения, при фиксированном $Y$ величина $h^{+} \omega^{+2}$ не зависит от $h^{+}$. Рис. 3 показывает, что независимость $h^{+} \omega^{+2}$ от $h^{+}$в первом приближении имеет место не только в логарифмическом пограничном слое, но и во всей внешней области вплоть до оси канала. Прямая линия построена по формуле (3), где принято значение постоянной Кармана 0.4. Зависимость $h^{+} \omega^{+2}$ от $h^{+}$наблюдается, но сравнительно слабая, т.е. кривые располагаются близко одна от 


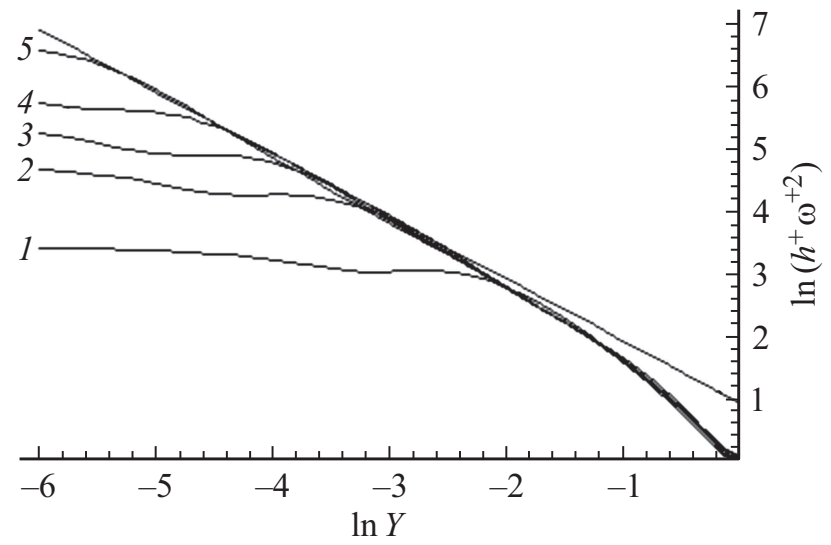

Рис. 3. Зависимости $\ln \left(h^{+} \omega^{+2}\right)$ от $\ln Y$ при различных значениях $h^{+}$. Кривые построены по результатам работы [16]. Кривые 1-5 построены при значениях $h^{+} 182,543,1001,1995$ и 5186 соответственно. Прямая линия представляет собой зависимость (3) в логарифмических координатах при $\kappa=0.4$.

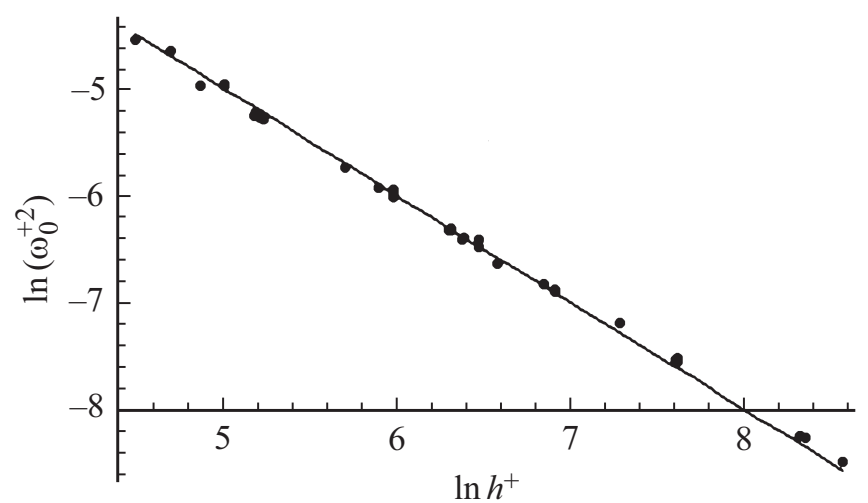

Рис. 4. Зависимость $\ln \left(\omega_{0}^{+2}\right)$ от $\ln h^{+}$. Точки построены по результатам работ [8-16], прямая - по формуле (4).

другой. Эта закономерность нарушается вблизи стенки. Разумеется, отклонения начинаются при тем бо́льших значениях $Y$, чем меньше $h^{+}$.

Соотношение (3) не может выполняться в окрестности оси канала. Слагаемое в уравнении баланса кинетической энергии турбулентных пульсаций, которое описывает обмен энергией между осредненным движением и турбулентными пульсациями, на оси канала обращается в нуль и в окрестности оси является малым. В этой области главные слагаемые - диссипация энергии в единице объема и слагаемое, описывающее перенос энергии за счет пульсаций давления. Заметим, что в силу симметрии производная по $Y$ от $\omega^{+2}$ на оси канала должна обращаться в нуль. Поэтому формулу (3) следует модифицировать таким образом, чтобы это условие выполнялось. Кроме того, при $Y=1$ значение $h^{+} \omega^{+2}$ отличается от $1 / \kappa$. Обозначим значение $\omega^{+}$на оси канала через $\omega_{0}^{+}$. Как видно из рис. 4 , зависимость $\omega^{+2}$ от $h^{+}$ в логарифмических координатах близка к линейной и неплохо описывается при помощи формулы

$$
\omega_{0}^{+2}=\frac{1}{h^{+}} .
$$

Можно предложить и более громоздкое соотношение, которое лучше описывает результаты работ [8-16], например, такого вида:

$$
\ln \omega_{0}^{+2}=-0.345+\frac{0.0564}{\ln h^{+}-3.94}-0.949 \ln h^{+} .
$$

В силу симметрии вблизи оси канала при малых значениях $1-Y$ должно выполняться соотношение

$$
h^{+}\left(\omega^{+2}-\omega_{0}^{+2}\right)=a(1-Y)^{2} .
$$

Анализ результатов работ [8-16] показывает, что коэффициент пропорциональности $a$ в этом равенстве является функцией $h^{+}$. Он увеличивается при сравнительно малых значениях $h^{+}$, достигает максимума примерно при $h^{+}=1000$ и затем медленно убывает. Среднее значение этой величины равно 7.54. В результате вместо соотношения (3) можно предложить следующую формулу:

$$
h^{+} \omega^{+2}=\frac{2.5}{Y}-1.5 Y+2.5 \ln Y+6.3(1-Y)^{2} .
$$

При малых значениях $Y$ эта формула переходит в (3), а при малых значениях $1-Y$ согласуется с формулами (4) и (5).

Так как $h^{+} \omega^{+2}$, вообще говоря, зависит от $h^{+}$, лучшее приближение можно получить, если использовать следующее выражение:

$$
h^{+}\left(\omega^{+2}-\omega_{0}^{+2}\right)=b\left(\frac{1}{Y}-1+\ln Y\right)+c(1-Y)^{2},
$$

где коэффициенты $b$ и $c$ рассматриваются как функции $h^{+}$. При помощи данных работ $[8-14,16]$ для них можно предложить следующие формулы:

$$
\begin{gathered}
b=2.404+\frac{115.2}{h^{+}}, \\
c=5.557+\frac{427.3}{h^{+}}-\frac{127.8 \ln h^{+}}{h^{+}} .
\end{gathered}
$$

Рассмотрим среднее по сечению канала значение квадрата модуля пульсационной составляющей вихря скорости $\omega_{m}^{+2}$. Эта величина определяется при помощи следующего равенства:

$$
\omega_{m}^{2+}=\int_{0}^{1} \omega^{2+} d Y .
$$

Величина $h^{+} \omega_{m}^{+2}$ при увеличении $h^{+}$числа заметно увеличивается. Для расчета $\omega_{m}^{+2}$ можно предложить следующее равенство:

$$
\ln \omega_{m}^{+2}=2.718-\frac{4.3}{\ln h^{+}-1.822}-0.9257 \ln h^{+} .
$$




\section{Распределение пульсационной составляющей вихря скорости В окрестности стенки}

Если во внешней области среднеквадратичные значения проекций пульсационной составляющей вихря скорости слабо различаются между собой, то в окрестности стенки это не так. О преимущественной ориентации пульсационной составляющей вихря скорости можно судить по численным значениям величин: $\omega_{x}^{2} / \omega^{2}, \omega_{y}^{2} / \omega^{2}$ и $\omega_{z}^{2} / \omega^{2}$. Так, величина $\omega_{x}^{2} / \omega^{2}$ представляет собой среднее значение квадрата косинуса угла, который образует пульсационная составляющая вихря скорости с осью $x$ и т. п. На стенке величина $\omega_{y}$ обращается в нуль и мала в окрестности стенки. Как ясно из рис. 5 , наибольшее численное значение вблизи стенки имеет величина $\omega_{z}^{2} / \omega^{2}$. Вблизи стенки эта характеристика увеличивается с ростом $y^{+}$и достигает максимального значения в вязком подслое на небольшом расстоянии $p_{1 z}^{+}$от стенки. Эта величина слабо зависит от $h^{+}$. При помощи данных работ $[8-14,16]$ можно получить формулу для ее расчета

$$
p_{1 z}^{+}=2.676+\frac{6.351}{h^{+}}-\frac{41.98 \ln h^{+}}{h^{+}}+\frac{6.856\left(\ln h^{+}\right)^{2}}{h^{+}} \text {. }
$$

При дальнейшем увеличении $y^{+}$величина $\omega_{z}^{2} / \omega^{2}$ уменьшается и на некотором расстоянии до стенки $p_{2 z}^{+}$принимает наименьшее значение. Это расстояние также зависит от $h^{+}$. Значения $p_{2 z}^{+}$, определенные по данным работ $[8-14,16]$, сильно различаются. В среднем эта величина составляет примерно 85 . Во внешней области $\omega_{z}^{2} / \omega^{2}$ не остается постоянной, но сравнительно медленно увеличивается.

Обозначим через $\alpha_{0}$ значение $\omega_{z}^{2} / \omega^{2}$ на стенке канала, через $\alpha_{1}$ максимальное значение этой величины, а через $\alpha_{2}$ - минимальное. Первые две характеристики уменьшаются при увеличении числа Рейнольдса и могут

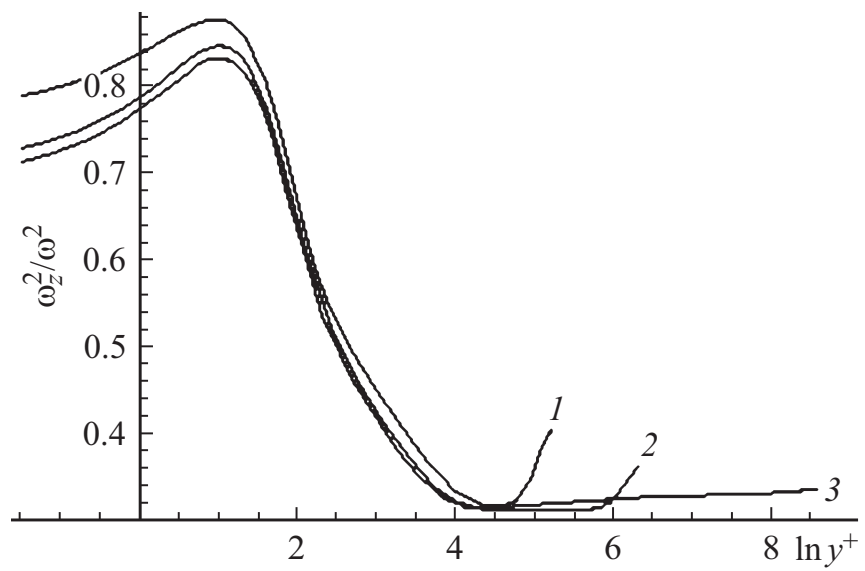

Рис. 5. Зависимости $\omega_{z}^{2} / \omega^{2}$ от $\ln y^{+}$. Кривые 1-3 построены при значениях 182, 543 и 5186 соответственно по результатам работы [16].

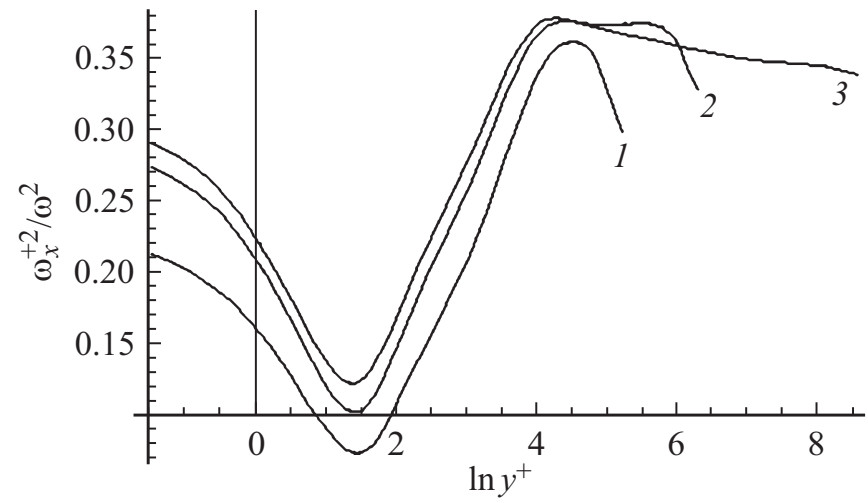

Рис. 6. Зависимости $\omega_{x}^{2} / \omega^{2}$ от $\ln y^{+}$. Кривые $1-3$ построены при значениях $h^{+} 182,543$ и 5186 соответственно по результатам работы [16].

быть найдены по формулам

$$
\begin{gathered}
\alpha_{0}=0.699-\frac{54.06}{h^{+}}+\frac{30.14 \ln h^{+}}{h^{+}}-\frac{3.325\left(\ln h^{+}\right)^{2}}{h^{+}}, \\
\alpha_{1}=0.8289+\frac{6.024}{h^{+}}+\frac{0.3999 \ln h^{+}}{h^{+}},
\end{gathered}
$$

Зависимость $\alpha_{2}$ от $h^{+}$по данным работ $[8-14,16]$ не является монотонной. Аппроксимационная формула имеет вид

$$
\alpha_{2}=0.313+\frac{3566}{h^{+1.87}}+\frac{2155 \ln h^{+}}{h^{+1.87}} .
$$

Величина $\omega_{x}^{2} / \omega^{2}$ в пределах вязкого подслоя убывает, достигает минимального значения, затем увеличивается и достигает максимума (рис. 6). Во внешней области наблюдается медленное уменьшение этой характеристики. Как координаты точек, где достигаются экстремумы, так и сами экстремальные значения зависят от $h^{+}$. Координата $p_{1 x}^{+}$точки, где достигается минимум, с ростом $h^{+}$слегка уменьшается и может быть вычислена по формуле:

$$
p_{1 x}^{+}=3.516+\frac{3.284}{\ln h^{+}} .
$$

Расстояние $p_{2 x}^{+}$до стенки, где достигается максимальное значение, можно рассчитать при помощи следующего соотношения:

$$
\begin{aligned}
p_{2 x}^{+} & =72.89+\frac{51.83}{\exp \left[3.08\left(\ln h^{+}-5.68\right)^{2}\right]} \\
& -\frac{3.205 \ln h^{+}}{\exp \left[3.08\left(\ln h^{+}-5.68\right)^{2}\right]}
\end{aligned}
$$

Обозначим через $\beta_{0}$ значение $\omega_{x}^{2} / \omega^{2}$ на стенке канала, через $\beta_{1}$ - минимальное значение этой величины, а через $\beta_{2}-$ максимальное. Все эти характеристики $h^{+}$ 


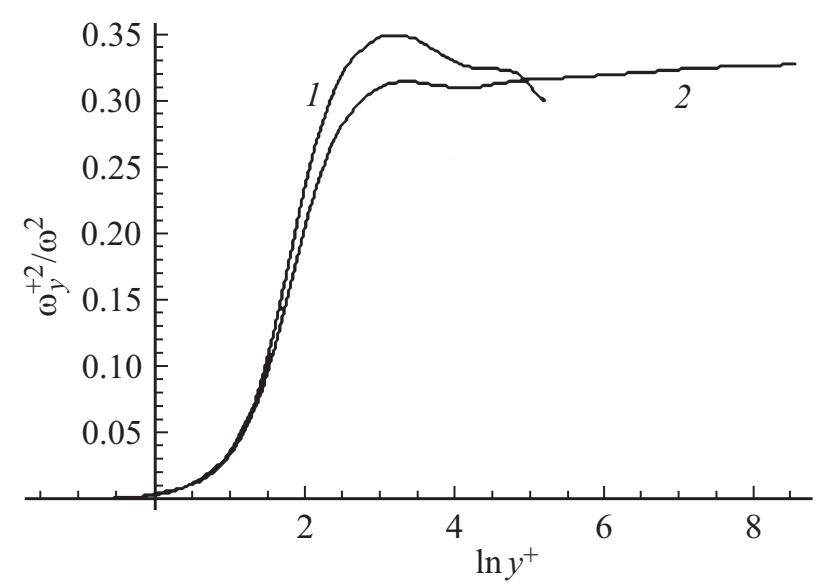

Рис. 7. Зависимости $\omega_{y}^{2} / \omega^{2}$ от $\ln y^{+}$. Кривые 1 и 2 построены при значениях $h^{+}=182$ и 5186 соответственно по результатам работы [16].

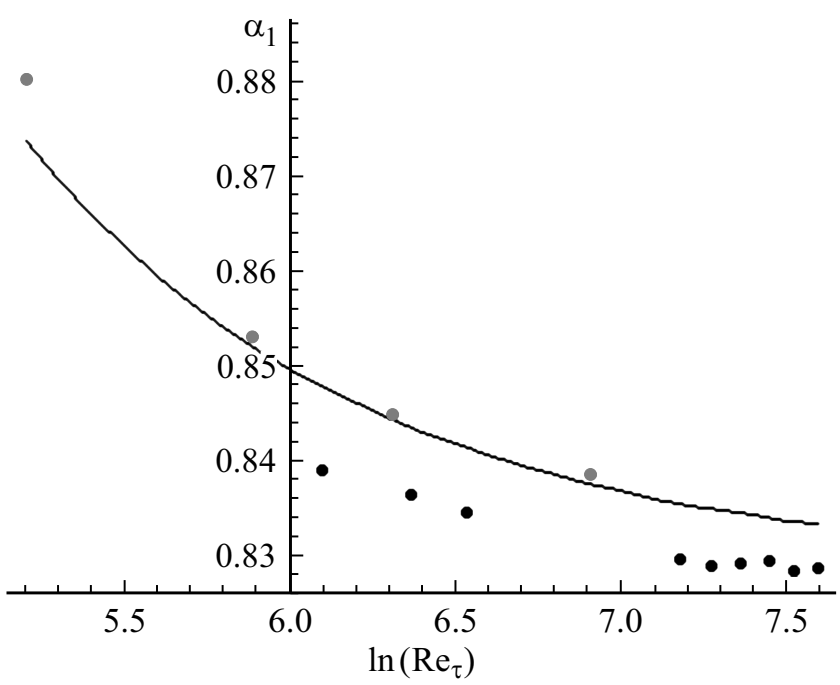

Рис. 8. Зависимость $\alpha_{1}$ от $\operatorname{Re}_{\tau}$ для течения в трубе и пограничном слое без градиента давления. Светлые точки построены по результатам работы [23], черные - по результатам работ $[24,25]$, кривая - по формуле (6).

с ростом $h^{+}$увеличиваются и могут быть рассчитаны по формулам

$$
\begin{gathered}
\beta_{0}=0.3135-\frac{29.3}{h^{+}}+\frac{2.832 \ln h^{+}}{h^{+}}, \\
\beta_{1}=0.133-\frac{14.32}{\left(\ln h^{+}\right)^{3.32}}, \\
\beta_{3}=0.377-\frac{6083}{h^{+2.47}} .
\end{gathered}
$$

Вблизи стенки величина $\omega_{y}^{2} / \omega^{2}$ зависит от $h^{+}$весьма слабо за исключением сравнительно малых значений $h^{+}$. Поэтому на рис. 7 показаны только два графика. При малых расстояниях до стенки рассматриваемая величина увеличивается и на некотором расстоянии до стенки $p_{y}^{+}$ достигается локальный максимум. Это расстояние и величина локального максимума $\gamma$ могут быть вычислены по формулам

$$
\begin{gathered}
p_{y}^{+}=31.14-\frac{231.1}{\left(\ln h^{+}\right)^{2.18}}, \\
\gamma=0.3132+\frac{8.628}{h^{+}}-\frac{0.407 \ln h^{+}}{h^{+}} .
\end{gathered}
$$

Можно ожидать, что для турбулентных течений вблизи твердой стенки другого типа, например, турбулентного течения в трубе и турбулентного течения в пограничном слое без градиента давления, в окрестности стенки результаты должны иметь сходный характер. Однако, как показывает рис. 8, имеются определенные количественные различия. Здесь в качестве определяющего линейного размера при расчете $\operatorname{Re}_{\tau}$ для течения в трубе используется радиус трубы, для течения в пограничном слое - его толщина. В роли $\omega_{z}$ для течения в трубе выступает тангенциальная составляющая вихря скорости. При построении рисунка использовались результаты прямого численного моделирования, полученные в работах [23-25]. В качестве иллюстрации здесь построена зависимость определенной выше величины $\alpha_{1}$ от критерия Рейнольдса $\operatorname{Re}_{\tau}$, рассчитанного по динамической скорости. Результаты, полученные для течения в трубе в работе [23], хорошо согласуются с формулой (6). Однако для течения в пограничном слое [24,25] эта характеристика принимает заметно меньшие значения.

\section{Заключение}

Полученные в работе результаты показывают, что имеется существенная анизотропия распределения пульсационной составляющей вихря скорости в области течения вблизи стенки, однако эта анизотропия сосредоточена в сравнительно узкой области. В первом приближении расстояния до стенки, где сильно различаются проекции вихря скорости, в переменных стенки не превосходят 100. Соответственно толщина этой области, отнесенная к полуширине канала, при увеличении числа Рейнольдса $\operatorname{Re}_{\tau}$ уменьшается обратно пропорционально этому числу. Получены формулы для расчета некоторых характеристик распределения завихренности. Их можно использовать в следующем диапазоне значений числа Рейнольдса: $109<\operatorname{Re}_{\tau}<5186$.

\section{Список литературы}

[1] Wallace J.M., Foss J. // Annu. Rev. Fluid. Mech. 1995. Vol. 27. P. 469-514.

[2] Kastrinakis E.G., Eckelmann H. // J. Fluid. Mech. 1983. Vol. 137. P. 165-186.

[3] Balint J.-L., Wallace J.M., Vukoslavčević P. // J. Fluid. Mech. 1991. Vol. 228. P. 53-86.

[4] Rajagopalan S., Antonia R.A. // Exp. Fluid. 1993. Vol. 14. P. $142-144$. 
[5] Honkan A., Andreopoulos Y. // J. Fluid. Mech. 1997. Vol. 350. P. 29-96.

[6] Agui J.M., Andreopoulos Y. // Exp. Fluid. 2003. Vol. 34. P. $192-205$.

[7] Panton R.L. // Phys. Fluid. 2009. Vol. 21. P. 115104.

[8] Moser R.D., Kim J., Mansour N.N. // Phys. Flui. 1999. Vol. 11. N 4. P. 943-945.

[9] Iwamoto K., Suzuki S., Kasagi N. // Int. J. Heat Fluid. Flow. 2002. Vol. 23. P. 678-689.

[10] Hoyas S., Jiménez J. // Phys. Fluid. 2006. Vol. 18. P. 011702.

[11] Hoyas S., Jiménez J. // Phys. Fluid. 2008. Vol. 20. P. 101511.

[12] Wreman A.W., Kuerten J.G.M. // Phys. Fluid. 2014. Vol. 26. P. 015102.

[13] Wreman A.W., Kuerten J.G.M. // Phys. Fluid. 2014. Vol. 26. P. 085103.

[14] Lozano-Duran A., Jiménez J. // Phys. Fluid. 2014. Vol. 26. P. 011702.

[15] Bernardini M., Pirozzoli S., Orlandi P. // J. Fluid. Mech. 2014. Vol. 742. P. 171-191.

[16] Lee M., Mozer R.D. // J. Fluid. Mech. 2015. Vol. 774. P. 395415.

[17] Buschmann M., Gad-el-Hak M. // Physica D. 2010. Vol. 239. P. 1288-1295.

[18] Чесноков Ю.Г. // ЖТФ. 2010. Т. 80. Вып. 1. С. 33-39.

[19] Чесноков Ю.Г. // ЖТФ. 2011. Т. 81. Вып. 1. С. 30-34.

[20] Чесноков Ю.Г. // Изв. СПбГТИ (ТУ). 2016. № 36. С. $104-$ 107.

[21] Antonia R.A., Rajagopalan S., Zhu Y. // Exp. Fluid. 1996. Vol. 20. P. 393-394.

[22] Ландау Л.Д., Лифиии Е.М. Механика сплошных сред. М.: ГИТТЛ, 1955. $788 \mathrm{c.}$

[23] El Khouri G.K., Schlatter P., Noorani A., Fischer P.F., Brethouwer G., Johanson A.V. // Flow Turbul. Combust. 2013. Vol. 91. P. 475-495.

[24] Jimenéz J., Hoyas S., Simens M.P., Mizuno Y. // J. Fluid. Mech. 2010. Vol. 657. P. 335-360.

[25] Sillero J.A., Jimenéz J., Moser R.D. // Phys. Fluid. 2013. Vol. 25. P. 105102. 